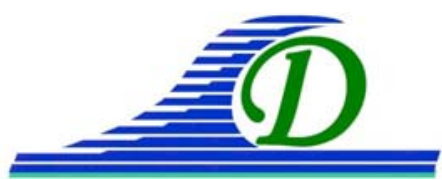

XIII ${ }^{\text {èmes }}$ Journées Nationales Génie Côtier - Génie Civil

Dunkerque, 2-4 juillet 2014

DOI:10.5150/jngcgc.2014.001 C Editions Paralia CFL

disponible en ligne - http://www.paralia.fr - available online

\title{
Propriétés statistiques des événements de tempête au large de la côte Basque
}

\author{
Stéphane ABADIE ${ }^{1,4}$, Michael GARDNER ${ }^{1,4}$, Philippe MARON $^{1,4}$, \\ Inaki DE SANTIAGO ${ }^{1,2}$, Denis MORICHON ${ }^{1,4}$, Noëlle BRU ${ }^{3,4}$
}

1. Laboratoire SIAME, EA4581, Université de Pau et des Pays de l'Adour, Allée du parc Montaury, 64600 Anglet, France.

stephane.abadie@univ-pau.fr

2. AZTI-tecnalia, Herrera Kaia, Portualdea z/g, E-20110 Pasaia, Gipuzkoa, Spain.

3. LMAP, UMR 5142, Université de Pau et des Pays de l'Adour, Avenue de l'Université, BP1155, 64013 Pau, France.

4. Station Marine d'Anglet, Fédération MIRA FR2152, Observatoire Aquitain des Sciences de l'Univers, Université de Pau et des Pays de l'Adour, Allée du parc Montaury, 64600 Anglet, France.

\section{Résumé :}

Dans ce travail, nous proposons une étude statistique des vagues de tempête sur la côte basque française et espagnole à partir d'un jeu de données issu de simulations numériques. Ce jeu de données, hébergé sur le site des ports espagnols (Puertos del Estado), couvre la période 1958 à nos jours. Après avoir validé ces données en comparaison de nos propres mesures, nous avons fait apparaître les événements de tempêtes, définis comme les événements extrêmes en termes d'énergie totale (i.e. l'intégrale du flux entre le début et la fin de la tempête). Puis nous avons analysé statistiquement les données obtenues et déterminé une classification permettant de dégager des groupements représentatifs de ces événements de tempête.

Mots-clés : États de mer, Événements de tempête, Analyse statistique, Classification, Simulation numérique.

\section{Introduction}

Dans le contexte actuel de changement climatique associé à une augmentation des enjeux sur le littoral (population, infrastructures, etc.), le suivi, la compréhension, la prédiction des événements de tempête revêt une grande importance. Le climat de houle au large de la côte basque est connu pour être particulièrement énergétique. Si l'on a une certaine connaissance de ce climat (e.g., ABADIE et al., 2006), l'analyse à proprement parlé des événements de tempête n'a pas encore été réalisée dans cette zone ni plus largement au niveau du territoire français. Le travail développé dans cet article est une première tentative pour combler cette lacune. 


\section{Présentation des données et méthode d'analyse}

\subsection{Données utilisées}

Les données utilisées dans ce travail sont principalement issues de la base de données SIMAR44 disponible à la demande sur le site http://www.puertos.es/. Ce jeu de données couvre la période 1958-2001. Il a été obtenu par simulation numérique avec une version avancée du modèle spectral WAM (WAMDI GROUP, 1988). Pour ces simulations, les termes de forçage proviennent d'une procédure de descente d'échelle effectuée à l'aide du modèle régional REMO (JACOB \& PODZUN, 1997) à partir des données atmosphériques globales du National Center for Environmental Prediction (NCEP). La résolution finale des données SIMAR est $1 / 24^{\text {ème }}$ de degré en spatial. A partir de 2001, les données proviennent de la base de données WANA, également disponible sur le site des ports espagnols. Ces données proviennent également de simulations à partir du code WAM mais sur une grille spatiale de $0.125^{\circ} \times 0.125^{\circ}$. Les données distribuées par les ports espagnols sont échantillonnées toutes les $3 \mathrm{~h}$. A des fins de comparaison, nous avons également utilisé les données mesurées par la bouée directionnelle DATAWELL située au large (partenariat UPPA / CEREMA, http://candhis.cetmef.developpementdurable.gouv.fr/) et dont les premières données remontent à 2009. La figure 1 présente l'emplacement géographique des points considérés dans cette étude. La profondeur associée aux points de donnée est (respectivement de A à E) : 165, 248, 140, 111, 40 m. La bouée ("Anglet" sur la figure 1) est mouillée dans $50 \mathrm{~m}$ de profondeur.

Les données de vague utilisées dans cet article sont :

- la hauteur significative spectrale: $H_{s}=H_{m 0}$

- la période de pic $T_{p}$ à partir de laquelle nous déduisons la période moyenne énergétique $T_{e}$ par le calcul approximatif suivant (CIRIA, 2007) $: T_{e}=T_{p} / 1.1$

- et enfin l'incidence moyenne du spectre $\theta_{m}$

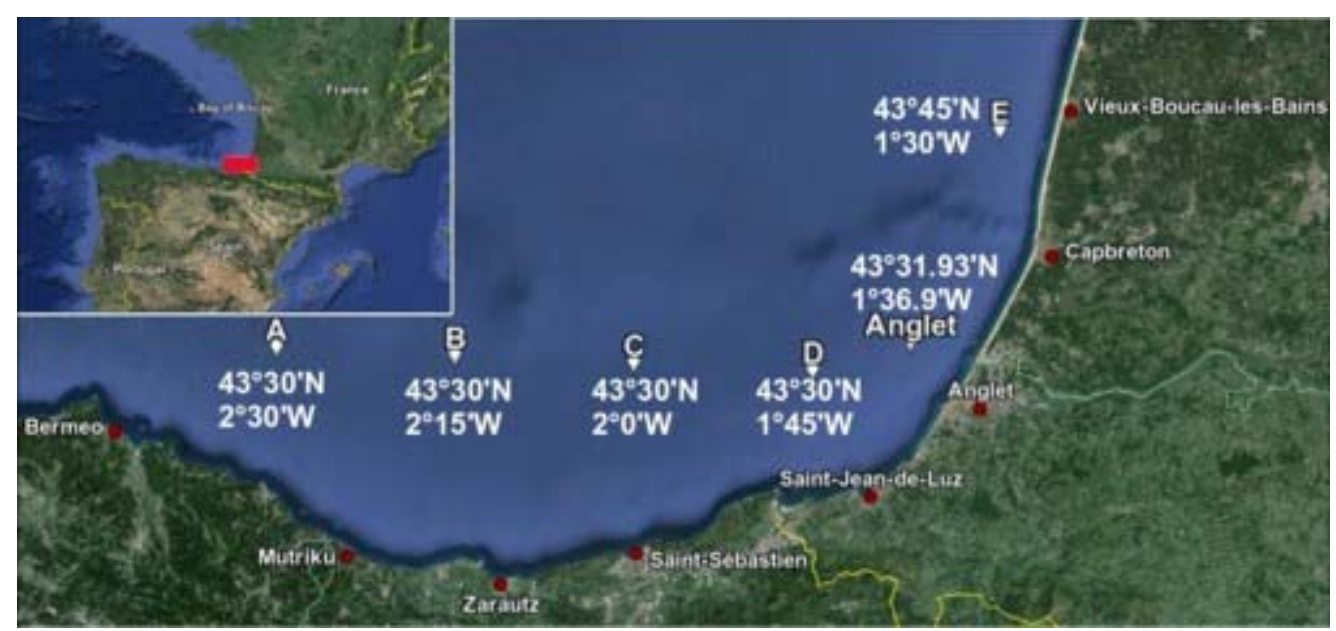

Figure 1. Localisation géographique de la zone d'étude et coordonnées des points de données utilisées pour l'analyse statistique. 


\section{XIII ${ }^{\text {èmes }}$ Journées Nationales Génie Côtier - Génie Civil \\ Dunkerque, 2-4 juillet 2014}

La consistance des données a été vérifiée en comparant les données de la base WANA au point $\mathrm{D}$ et la mesure par la bouée d'Anglet sur la période de recouvrement (i.e., septembre 2009 à mi-janvier 2013). Les hauteurs significatives de vagues sont correctement prédites par le modèle (coefficient de détermination $R^{2}=0.88$, hauteur significative moyenne de $1.63 \mathrm{~m}$ au point de mesure contre $1.57 \mathrm{~m}$ pour la simulation). En revanche, et comme on pouvait s'y attendre, les périodes de pics simulées sont légèrement moins en accord avec la mesure $\left(R^{2}=0.71\right)$.

\subsection{Méthodologie d'analyse}

Les tempêtes correspondent à des événements rares et de forte intensité. Classiquement, on considère les $10 \%$ des événements ayant le plus fort $H_{s}$ comme des tempêtes (e.g., DOLAN \& DAVIS, 1992 ; RANGEL-BUITRAGO \& ANFUSO, 2011). Cette valeur de $10 \%$ est arbitraire. On doit pouvoir l'affiner en mettant en regard les effets réels de ces événements à la côte mais ceci nécessite un retour d'expérience important que nous n'avons pas encore. En revanche, le choix de $H_{s}$ comme indicateur nous semble discutable. Nous avons choisi de travailler plutôt sur le flux d'énergie $F$ (en J/s par m de crête de vague ou $\mathrm{W} / \mathrm{m}$ ) estimé à partir des paramètres spectraux (CRUZ, 2008) qui nous semble être l'indicateur pertinent :

$$
F=\frac{1}{16} \rho g H_{s}^{2} C_{g}
$$

où $C_{g}$ est la vitesse de groupe évaluée à partir de la période énergétique moyenne $T_{e}$ et de la relation de dispersion, $g$ l'accélération de la pesanteur $\left(9.81{\mathrm{~m} . \mathrm{s}^{-2}}^{-2}\right.$ et $\rho$ la densité de l'eau de mer. Néanmoins, il faut souligner que la différence obtenue en prenant $H_{s}$ comme indicateur plutôt que le flux reste anecdotique. Nous avons ainsi calculé le flux d'énergie toutes les $3 \mathrm{~h}$ et en tous les points étudiés et retenu en chaque point les $10 \%$ des événements ayant les valeurs de flux les plus grandes. Cela nous permet de définir une valeur seuil du flux au-dessus de laquelle l'événement instantané est considéré comme faisant partie d'une tempête (figure 2). L'intensité d'une tempête particulière est ensuite déterminée en calculant le flux total entre le début et la fin de la tempête suivant (par une approximation de Simpson) :

$$
E=\int_{t_{d}}^{t_{f}} F(t) d t
$$

Chaque tempête indicée $i$, est ainsi définie par une intensité $E_{i}$ associée qui correspond à l'énergie totale mobilisée par cette tempête par mètre linéaire, et une durée $D_{i}$.

Notons que les tempêtes séparées de moins de $24 \mathrm{~h}$ sont regroupées car elles sont considérées comme faisant partie d'un même événement dépressionnaire. Pour chaque événement de tempête, on calcule également les valeurs moyennes des paramètres de vague : $H_{S_{i}}, T_{\theta_{i}}, \hat{\theta}_{i}$. 


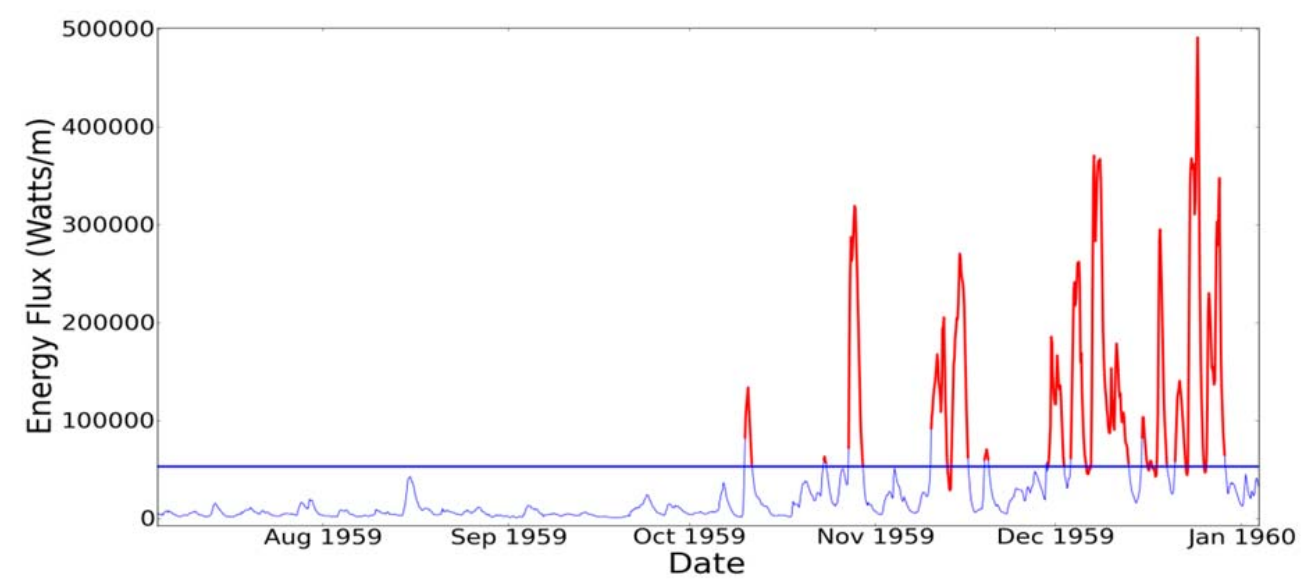

Figure 2. Valeur seuil du flux d'énergie au point A (61 kW/m) pour l'année 1959 avec en rouge les événements de tempête.

\section{Résultats}

\subsection{Analyse statistique}

Les seuils de flux de tempête varient de 55 à $70 \mathrm{~kJ} \mathrm{~m}^{-1} \mathrm{~s}^{-1}$ en augmentant graduellement de A vers E. Les données moyennes (notées "<>") et déviations standards (notées " $\sigma$ ") correspondant aux événements de tempêtes sont présentées dans le tableau 1. Les caractéristiques des événements de tempête varient peu entre la partie sud de la côte basque espagnole et nord de la côte basque française. Elles sont légèrement plus énergétiques dans la zone française. On observe en fait une augmentation graduelle de l'énergie d'une tempête moyenne de $\mathrm{A}$ vers $\mathrm{E}$. L'énergie dissipée durant une tempête moyenne caractéristique de cette zone se situe aux alentours de $18 \mathrm{GJ} / \mathrm{m}$ avec une déviation standard importante de l'ordre de $30 \mathrm{GJ} / \mathrm{m}$ et la durée moyenne de l'événement est légèrement inférieure à deux jours. Les vagues associées à ces événements moyens sont de l'ordre de $3.80 \mathrm{~m}$ et ont une période moyenne énergétique de l'ordre de $12.5 \mathrm{~s}$. La direction moyenne des tempêtes se situe aux alentours de $305^{\circ}$ avec une faible variabilité (déviation standard de l'ordre de $10^{\circ}$ sauf au large de Bilbao) caractéristique de la zone. Le point $\mathrm{E}$ moins profond est vraisemblablement soumis à la réfraction, ce qui peut expliquer l'écart en termes de direction moyenne observée. Avec une direction incidente de l'ordre de $300^{\circ}$, les houles de tempête arrive frontalement à Anglet dont le trait de côte est quasiment perpendiculaire à cette direction tandis qu'elles ont une incidence très forte vues du côté espagnol.

L'histogramme de la durée des tempêtes est relativement similaire en chaque point. Il est présenté au point $\mathrm{D}$ dans la figure 3 . On peut remarquer que $90 \%$ des tempêtes durent moins de 4 jours. Il existe néanmoins des événements durant plus de 15 jours et il serait intéressant de déterminer la période de retour de tels événements extrêmes. 


\section{XIII ${ }^{\text {èmes }}$ Journées Nationales Génie Côtier - Génie Civil \\ Dunkerque, 2-4 juillet 2014}

Tableau 1. Données moyennes et déviations standards des événements de tempêtes aux points étudiés.

\begin{tabular}{|c|c|c|c|c|c|}
\hline Variable/Point & A & B & C & D & E \\
\hline nombre d'événements & 1185 & 1219 & 1244 & 1214 & 1207 \\
\hline$\langle E>(G J / m)$ & 16,9 & 17,3 & 18,1 & 18,7 & 20,6 \\
\hline$\sigma(E)(G J / m)$ & 27,8 & 27,3 & 28,8 & 29,3 & 32,5 \\
\hline$<D>(H)$ & 43,5 & 42,9 & 42,9 & 42,9 & 43,3 \\
\hline$\sigma(D)(H)$ & 50,1 & 47,8 & 48,9 & 48,3 & 52,9 \\
\hline$\left\langle\hat{H}_{s}>(\mathrm{m})\right.$ & 3,70 & 3,81 & 3,84 & 3,85 & 3,88 \\
\hline$\sigma\left(\hat{H}_{s}\right)(\mathrm{m})$ & 0,68 & 0,69 & 0,70 & 0,71 & 0,70 \\
\hline$\left\langle\hat{T}_{e}\right\rangle(\mathrm{s})$ & 12,43 & 12,51 & 12,62 & 12,69 & 12,41 \\
\hline$\sigma\left(T_{e}\right)(\mathrm{s})$ & 1,98 & 1,95 & 1,90 & 1,86 & 1,88 \\
\hline$\left\langle\hat{\theta}_{m}>\left(^{\circ}\right)\right.$ & 307,71 & 306,57 & 305,10 & 303,86 & 295,28 \\
\hline$\sigma\left(\hat{\theta}_{m}\right)\left({ }^{\circ}\right)$ & 16,66 & 10,85 & 10,36 & 8,23 & 8,08 \\
\hline
\end{tabular}

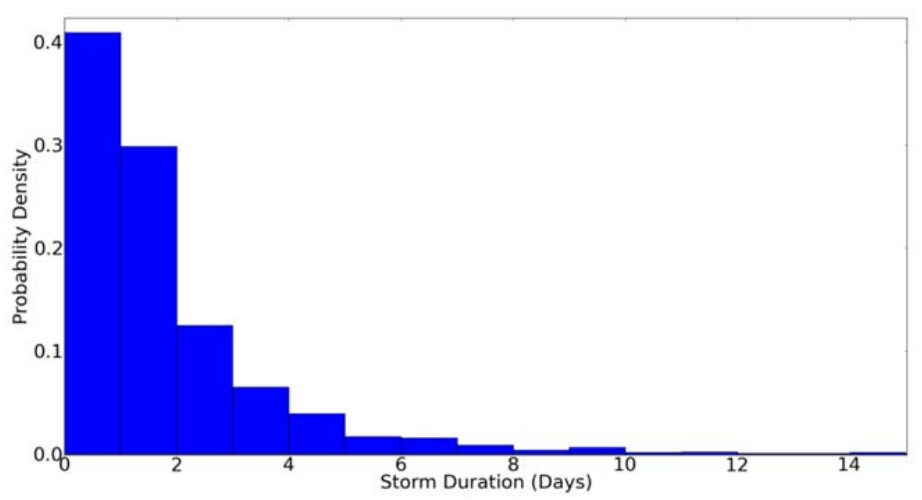

Figure 3. Histogramme de la durée des tempêtes au point D.

La figure 4 présente l'histogramme de la durée moyenne entre les tempêtes en différents points. Ce paramètre est important car la réponse d'une plage à ces événements énergétiques est différente selon que ceux-ci sont rapprochés en temps ou non. La plage s'adapte en effet de manière dynamique aux changements de régime hydrodynamique et les variations temporelles du forçage ont donc un effet drastique sur son évolution. Les résultats de la figure 4 permettent de quantifier cet effet et pourront plus tard être utilisés pour étudier des scénarios d'érosion à long terme par exemple.

La figure 5 permet maintenant d'illustrer le lien entre hauteur et période de vague durant les tempêtes. L'échelle de couleur représente la densité de probabilité associée à chaque couple $\left(H_{s_{l}}, T_{e_{l}}\right)$. On rappelle qu'il s'agit des données de vagues moyenne associées à chaque événement de tempête. Les résultats sont relativement similaires pour les 3 points étudiés. Les événements les plus fréquents sont des tempêtes associant une houle de hauteur significative moyenne légèrement supérieure à $3 \mathrm{~m}$ à une période moyenne énergétique de $14 \mathrm{~s}$. Pour des hauteurs de vague plus importantes, curieusement, la période énergétique diminue ce qui est contre intuitif et ne correspond pas aux 


\section{Thème 1 - Hydrodynamique côtière}

statistiques des données brutes. Ce comportement provient peut-être d'un effet de lissage induit par l'opération de moyenne sur les tempêtes.

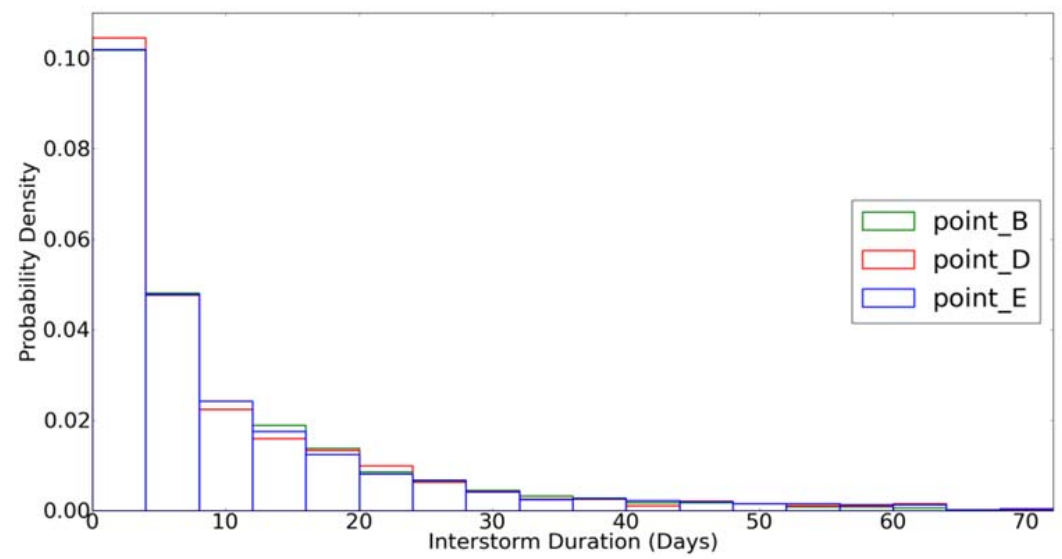

Figure 4. Histogramme de la durée inter-tempête aux points B, D et E.
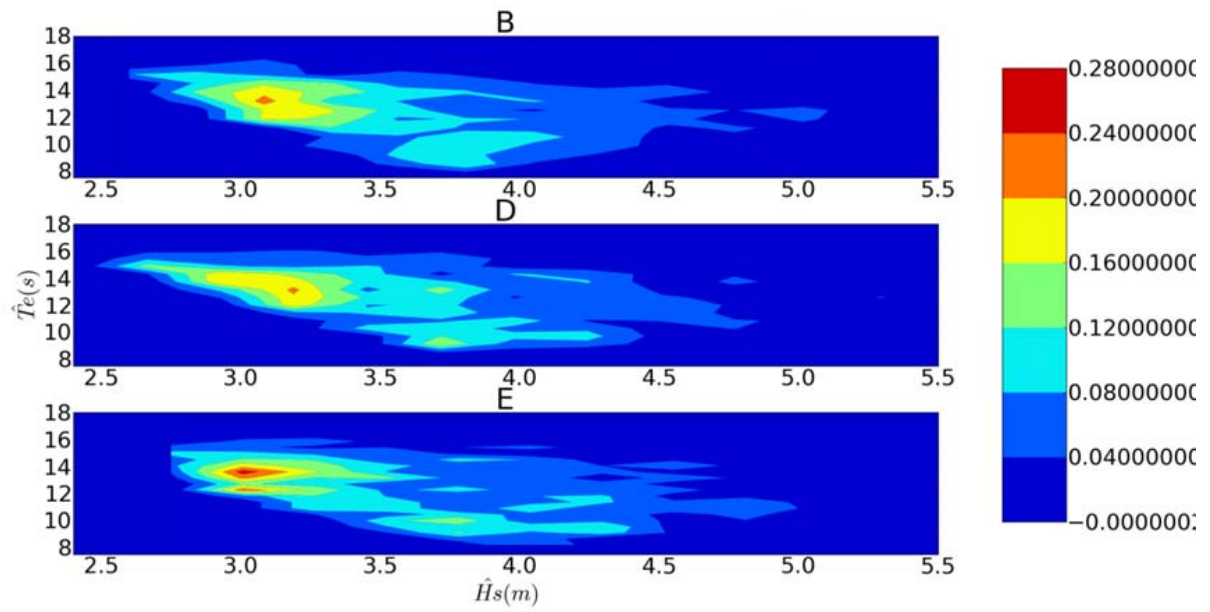

Figure 5. Histogramme bivarié de la hauteur significative et de la période énergétique (moyenne calculée sur chaque tempête) aux points $B, D$ et $E$.

\subsection{Classification}

L'analyse statistique est importante pour quantifier des tendances mais elle génère beaucoup d'information dont il peut être difficile d'extraire les paramètres significatifs. La classification des événements de tempête permet de réduire cette information en un nombre limité de paramètres qui vont ensuite pouvoir être utilisés de manière pratique (e.g., pour de la simulation numérique). Il s'agit d'une méthode d'analyse qui vise à diviser un ensemble de données en différents paquets homogènes. Nous avons réalisé une classification sur l'énergie totale de chaque tempête aux points B, D et E en utilisant la méthode hiérarchique. Cette méthode itérative revient à regrouper progressivement les points (ou clusters formés) 2 à 2 suivant un certain critère et en remplaçant le cluster 


\section{XIII ${ }^{\text {èmes }}$ Journées Nationales Génie Côtier - Génie Civil \\ Dunkerque, 2-4 juillet 2014}

ainsi généré par un centre équivalent. Dans cette étude nous avons utilisé la méthode de Ward (WARD, 1963) qui permet de trouver à chaque étape, l'agrégation qui minimise la perte d'information.

L'application de cette méthode sur l'énergie totale de chaque tempête donne 3 ou 4 clusters d'énergie totale de tempête suivant le point considéré. Ce nombre est un compromis trouvé en cherchant les clusters les plus séparés possibles tout en assurant la plus grande homogénéité à l'intérieur de chaque cluster. La première ligne du tableau 2 donne les classes trouvées au point $\mathrm{D}$ (i.e. au large d'Anglet), leur poids respectif et l'énergie associée aux centres de ces clusters.

Une même énergie totale de tempête peut être générée par différents triplets de hauteur significative, période moyenne énergétique et durée de tempête. Pour des besoins futurs de simulation numérique, nous avons besoin de disposer d'informations sur des sous classes de ce type. Pour chaque classe de tempête, nous avons donc effectué une sous classification à 2 paramètres. Le troisième paramètre qui est la durée de tempête se déduit en effet des deux autres de manière à trouver l'énergie totale de la classe de tempête concernée. Cette procédure nous donne entre 2 et 4 sous classes pour chaque classe de tempête (soit au total 34 sous classe pour l'ensemble des points étudiés (i.e. B, $\mathrm{D}$ et $\mathrm{E}$ )). Ces sous-classes sont également indiquées dans le tableau 2 pour le point $\mathrm{D}$.

Tableau 2. Résultat de la classification hiérarchique au point D.

\begin{tabular}{|c|c|c|c|c|c|c|c|c|c|c|}
\hline $\begin{array}{c}\text { Cluster (E) } \\
\text { (poids de } \\
\text { classe) }\end{array}$ & \multicolumn{3}{|c|}{$\begin{array}{c}1 \\
(0.244)\end{array}$} & \multicolumn{3}{|c|}{$\begin{array}{c}2 \\
(0.667)\end{array}$} & \multicolumn{4}{|c|}{$\begin{array}{c}3 \\
(0.089)\end{array}$} \\
\hline$<\mathrm{E}>(\mathrm{GJ} / \mathrm{m})$ & \multicolumn{3}{|c|}{27.86} & \multicolumn{3}{|c|}{5.37} & \multicolumn{4}{|c|}{94.02} \\
\hline $\begin{array}{l}\text { Sous-classe } \\
\text { (poids } \\
\text { de classe) }\end{array}$ & $\begin{array}{c}1 \\
(0.29)\end{array}$ & $\begin{array}{c}2 \\
(0.26)\end{array}$ & $\begin{array}{c}3 \\
(0.45)\end{array}$ & $\begin{array}{c}1 \\
(0.39)\end{array}$ & $\begin{array}{c}2 \\
(0.26)\end{array}$ & $\begin{array}{c}3 \\
(0.35)\end{array}$ & $\begin{array}{c}1 \\
(0.31)\end{array}$ & $\begin{array}{c}2 \\
(0.24)\end{array}$ & $\begin{array}{c}3 \\
(0.11)\end{array}$ & $\begin{array}{c}4 \\
(0.34)\end{array}$ \\
\hline$<\mathrm{H}_{\mathrm{s}}>(\mathrm{m})$ & 4.11 & 5.16 & 3.88 & 3.15 & 4.15 & 3.64 & 4.31 & 4.08 & 4.63 & 5.56 \\
\hline$<\mathrm{T}_{\mathrm{e}}>(\mathrm{s})$ & 12.21 & 11.96 & 14.55 & 14.27 & 9.91 & 12.02 & 13.59 & 4.86 & 12.04 & 13.69 \\
\hline
\end{tabular}

\section{Conclusions et perspectives}

Dans cette étude, nous avons analysé des simulations d'états de mer au large de la côte Basque couvrant une période allant de 1958 à nos jours. Nous avons extrait de ce jeu de données les événements de tempête caractérisés par le dépassement d'un seuil sur le flux d'énergie. Les caractéristiques moyennes des tempêtes au large de la côte basque sont: une énergie totale mobilisée d'environ $18 \mathrm{JG} / \mathrm{m}$ et une durée de 2 jours avec des vagues associées de hauteur significative moyenne $3.8 \mathrm{~m}$, de période énergétique moyenne $12.5 \mathrm{~s}$ et de direction moyenne de provenance de $305^{\circ}$ (très peu variable). Ces caractéristiques sont peu variables en espace (de Bilbao à Vieux Boucau). L'étude 
fournit également les distributions statistiques des différentes variables ainsi que des classes et sous classes correspondant à ces événements de tempêtes. L'ensemble de ces données pourra être utilisé pour mieux comprendre l'impact des tempêtes à la côte par le biais de la simulation par exemple.

\section{Remerciements}

Les auteurs de cet article remercient les ports espagnols (Puertos des Estado) pour avoir permis la mise à disposition des données SIMAR et WANA. Michael Gardner a bénéficié du programme d'échange entre l'Université de Rhode Island (USA) et l'Université Technologique de Compiègne. Ce travail est en partie le résultat de son stage de fin d'année effectué au laboratoire SIAME. Cette recherche a bénéficié des fonds Bonus Qualité Recherche de l'Université de Pau et des Pays de l'Adour.

\section{Références bibliographiques}

ABADIE S., BUTEL R., MAURIET S., MORICHON D., DUPUIS H. (2006). Wave climate and longshore drift on the South Aquitaine Coast. Continental Shelf Research, Vol. 26(16), pp 1924-1939. http://dx.doi.org/10.1016/j.csr.2006.06.005

CIRIA (2007). The rock manual, C683, The use of rock in Hydraulic Engineering, 1304 p, Construction Industry Research \& Information Association (CIRIA); 2nd edition.

CRUZ J. (2008). Ocean Wave Energy. Springer, 421 p.

DOLAN R., DAVIS R. (1992). An intensity scale for Atlantic Coast Northeast Storms. Journal of Coastal Research. Vol. 8(4), pp 840-853.

JACOB D., PODZUN R. (1997). Sensitivity study with the regional climate model REMO. Meteorol. Atmos. Phys., Vol. 63(1-2), pp 119-129. http://dx.doi.org/10.1007/BF01025368

RANGEL-BUITRAGO R., ANFUSO G. (2011). Coastal Storm Characterization and morphological impacts on sandy coasts. Earth Surface Processes and Landforms. Vol. 36(15), pp 1997-2010. http://dx.doi.org/10.1002/esp.2221

WAMDI GROUP (1988). The WAM model - A third generation ocean wave prediction model. Journal of Physical Oceanography, Vol. 18, pp 1775-1810. http://dx.doi.org/10.1175/1520-0485(1988)018<1775:TWMTGO>2.0.CO;2

WARD J.H. (1963). Hierarchical Grouping to Optimize an Objective Function, Journal of the American Statistical Association, Vol. 58, pp 236-244. http://dx.doi.org/10.1080/01621459.1963.10500845 